\title{
FURTHER STUDIES OF STERN WAVEMAKING
}

\author{
D. E. FARROW ${ }^{1}$ and E. O. TUCK ${ }^{2}$ \\ (Received 23 February 1993; revised 14 August 1993)
}

\begin{abstract}
A new numerical method is applied to the problem of inviscid irrotational flow past a semi-infinite stern-like body of general shape. Both smooth-detachment and stagnantdetachment flows are considered, in the context of varying the geometry of the stern to generate very small waves, with the eventual aim of eliminating waves altogether. The results of this work confirm previously published results for the smooth-detachment case, but cast doubt on the existence of waveless solutions for stagnant detachment.
\end{abstract}

\section{Introduction}

The energy contained in the surface waves generated by a moving ship is an important component of the overall drag on a ship. In two dimensions, the waves far behind a moving ship can be thought of, at least in a linear sense, as the superposition of separate bow and stern waves (see Tuck [3]). These waves will interfere destructively if they are out of phase by $180^{\circ}$, which will occur at special values of the length-based Froude number $F r_{L}=U /(g L)^{1 / 2}$, where $U$ is the speed of the ship, $g$ is gravity and $L$ is the ship's length. This qualitative result holds for many finite two-dimensional bodies, and is not restricted to ships. A pressure distribution (modelling a hovercraft) of finite length also generates no waves far downstream at special length-based Froude numbers [7].

The current work is concerned with the wavemaking of an isolated stern of finite draft $D$, the length $L$ being assumed sufficiently great so that the effect of the bow can be ignored. In particular, can the shape of the stern be modified so that there are no waves in the flow far downstream? Such a flow would be doubly useful since, owing to the quadratic nature of the nonlinearity, the flow could also be reversed to yield a splashless bow flow [3].

\footnotetext{
${ }^{1}$ School of Mathematics, University of East Anglia, Norwich NR4 7TJ, England.

${ }^{2}$ Department of Applied Mathematics, The University of Adelaide, South Australia 5005, Australia.

(C) Australian Mathematical Society, 1995, Serial-fee code 0334-2700/95
} 
Stern-like flows such as those described above have been considered before. Vanden-Broeck and Tuck [6] studied the two-dimensional flow past a semi-infinite stern-like body with a stagnation point where the free surface meets the body. In that paper, the semi-infinite body ends with a straight section at an arbitrary inclination. Vanden-Broeck and Tuck used divergent series summation techniques and numerical methods to obtain free surface profiles and relationships between the draft-based Froude number $\mathrm{Fr}=U /(g D)^{1 / 2}$ and the steepness of the downstream waves. More general stern geometries were examined by Vanden-Broeck and Tuck [4], with the explicit aim of finding sterns that generate no downstream waves. All the work mentioned above is for steady flow, unlike that of Yeung [8] who found numerical solutions for the start-up flow behind a semi-infinite stern-like body of arbitrary geometry.

Another possible flow configuration (other than the above type including a stagnation point) has the free surface detaching smoothly from the body at an idealised transom. In this case, the slope of the free surface matches that of the body, and the body is effectively "planing". Vanden-Broeck [5] analytically and numerically studied the flow past a semi-infinite flat plate with smooth detachment, and found that flows of this type existed only for sufficiently large draft-based Froude numbers. All these solutions possessed waves, although the magnitude of the waves decreased as the draft-based Froude number increased.

A different approach to seeking waveless solutions was used by Madurasinghe and Tuck [2] and Madurasinghe [1]. In those papers, the waveless nature of the solution is specified a priori, and a geometrical parameter is determined automatically as part of the solution, so as to guarantee this waveless property. The solutions presented in those papers will be discussed later in the context of the present results.

The approach taken in the present work is to calculate the free-surface profile (including waves) behind a general stern-like body, and then to adjust geometrical parameters to seek waveless solutions. This allows the nature of the solutions in the parametric neighbourhood of any waveless solutions to be examined.

(a)

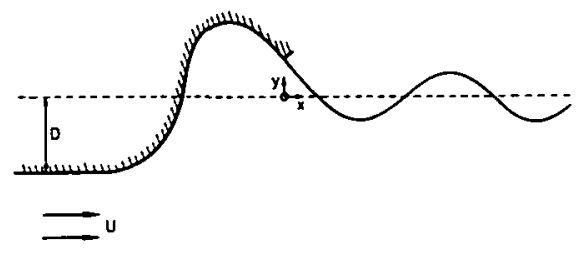

(b)

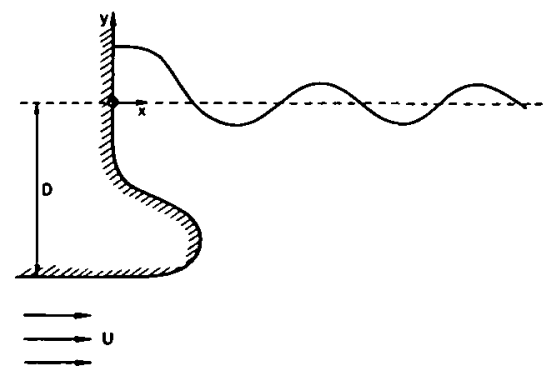

FIGURE 1. Definition sketch for the two flow configurations. (a) Smooth detachment. (b) Stagnant detachment. 


\section{Formulation}

Consider the steady two-dimensional irrotational flow of an incompressible inviscid fluid past a semi-infinite stern-like body. The geometries of the two types of flow examined in this paper are sketched in Figure 1. For the first type, the slope of the free surface at the point of attachment matches that of the body (Figure 1(a)), while the second type has a stagnation point at attachment (Figure 1(b)). Suppose that the ship has a flat horizontal bottom $y=-D$ except for some finite region close to the stern, and that far from the free surface, the flow is a horizontal uniform stream of speed $U$.

Let the dimensional velocity potential be denoted by $\phi^{\prime}$ and the dimensional stream function by $\psi^{\prime}$. Suppose, without loss of generality, that the free surface and the body are given by $\psi^{\prime}=0$, and that $\phi^{\prime}=0$ at the point of attachment. Denote the value of $\phi^{\prime}$ at the point where the the body becomes horizontal (see figure 1 ) by $-K$. Following Tuck \& Vanden-Broeck [4], the system is nondimensionalised using the length scale $U / K$ and the velocity scale $U$.

Let $u-i v$ be the (nondimensional) complex velocity field associated with the complex potential $f=\phi+i \psi$. The function $\Omega=\tau-i \theta$ is defined by

$$
\frac{d f}{d z}=\exp (\tau-i \theta)
$$

and is thus an analytic function of $f$ in the half plane $\psi<0$. The quantity $\theta$ can be identified as the angle of the local fluid velocity vector to the horizontal, and $\tau$ as the logarithm of the magnitude of this velocity. The flow problem is solved in the inverse sense by finding $\Omega$ as a function of $f$ in the half plane $\psi<0$. The dynamic free surface condition is that the pressure is constant there, and hence by Bernoulli's equation

$$
\gamma y+\frac{1}{2} q^{2}=\text { constant on } \psi=0
$$

where $\gamma=g K / U^{3}$ is a nondimensional gravity. Differentiating (2) tangentially, that is, with respect to $\phi$, and noting that $q=\exp (\tau)$, yields

$$
\gamma \frac{\partial y}{\partial \phi}+\frac{1}{2} \frac{\partial}{\partial \phi} e^{\tau}=0
$$

From (1), $\partial y / \partial \phi=e^{-\tau} \sin \phi$, and since $\Omega(f)$ is an analytical function in the halfplane $\psi<0$, the value of $\tau$ on the free surface can be related to $\theta$ there via a Hilbert transform

$$
\tau(\phi, 0)=\frac{1}{\pi} f_{-\infty}^{\infty} \frac{\theta(\varphi, 0)}{\varphi-\phi} d \varphi
$$

where the integral is of Cauchy principal value form. Thus (3) can be written

$$
\gamma \sin \phi+\frac{1}{3} \frac{\partial}{\partial \phi} \exp \left(\frac{3}{\pi} f_{-\infty}^{\infty} \frac{\theta(\varphi, 0)}{\varphi-\phi} d \varphi\right)=0
$$


Integrating from the attachment point $\phi=0$ yields a nonlinear singular integral equation

$$
0=3 \gamma \int_{0}^{\phi} \sin \theta(\varphi) d \varphi+F(\phi) \exp \left(\frac{3}{\pi} f_{0}^{\infty} \frac{\theta(\varphi)}{\varphi-\phi} d \varphi\right)-q_{0}^{3}
$$

which applies on the free surface $\psi=0, \phi>0$. The function $F(\phi)$ given by

$$
F(\phi)=\exp \left(\frac{3}{\pi} f_{-1}^{0} \frac{\Theta(\varphi)}{\varphi-\phi} d \varphi\right)
$$

is a known function of $\phi$, since $\Theta(\varphi), \varphi<0$, is presumed known, and $q_{0}$ is the fluid speed at the point of attachment at $\phi=0$. For the flow with a stagnation point at attachment, $q_{0}=0$.

The aim here is to find some form for $\Theta(\varphi)$ in $-1<\varphi<0$ (and thus $F(\phi)$ in $\phi>0$ ) for which the solution of (6) has no waves, or at least very small waves, as $\phi \rightarrow+\infty$. If there are no waves, then $\theta \rightarrow 0$ as $\phi \rightarrow+\infty$ and a limit exists for large $\phi$ in (6). This limit yields an integral constraint that must hold for any waveless solution, namely

$$
0=1+3 \gamma \int_{0}^{\infty} \sin \theta(\varphi) d \varphi-q_{0}^{3}
$$

\section{Numerical method}

The unknown function in (6) is $\theta(\phi)$ for $\phi>0$. In the smooth-detachment case, the detachment velocity $q_{0}$ is also not known, but can be written in terms of $\theta$ via

$$
q_{0}=\exp \left(\frac{1}{\pi} f_{-1}^{\infty} \frac{\theta(\varphi)}{\varphi} d \varphi\right)
$$

The equation for $\theta$ is discretised by introducing the set of points $\phi_{1}, \ldots, \phi_{N}$ with the associated unknowns $\theta_{i}=\theta\left(\phi_{i}\right), i=1, \ldots, N$. The $\phi_{i}$ must be chosen so that the important behaviour of $\theta$ is resolved. If $\theta_{0}=\theta\left(0^{+}\right)$then for both flows, $\left(\theta-\theta_{0}\right) \propto \sqrt{\phi}$ as $\phi \rightarrow 0^{+}$, so near $\phi=0$, the $\phi_{i}$ are chosen to be

$$
\phi_{i}=i^{2} \Delta \phi_{1}, \quad i=1, \ldots, M,
$$

where $\Delta \phi_{1}$ is the smallest step size and $M$ is to be determined. For large $\phi, \theta$ is in general wavelike and the (linear theory) wavenumber is $\gamma$. To resolve these waves, $\Delta \phi_{i}=\phi_{i}-\phi_{i-1}$ is not permitted to increase beyond an upper limit $\Delta \phi_{\max }$ determined by the minimum number of mesh points per wavelength. Thus $M$ is given by

$$
M=\left[\frac{1}{2}+\frac{\Delta \phi_{\max }}{2 \Delta \phi_{0}}\right]
$$


and $\phi_{i}$ for $i>M$ is simply given by $\phi_{i}=\phi_{i-1}+\Delta \phi_{M}$, that is, a uniform grid. The cut-off point $\phi_{N}$ is determined by stipulating the number of waves within the computational domain, typically four or more. In the simulations described below, $\Delta \phi_{1} \approx 0.005$, and there are about 30 points per wavelength far from the stern.

With the discretisation now in place, (6) is now forced to hold at $\phi=\phi_{i}$ for $i=1, \ldots, N-1$, which yields $N-1$ nonlinear equations for the $N$ unknowns $\theta_{i}$, $i=1, \ldots, N$. The $N$ th equation is obtained by writing $\theta_{N}$ as a quadratic extrapolation of $\theta_{N-3}, \theta_{N-2}$ and $\theta_{N-1}$. In (6), $F(\phi)$ can be calculated exactly from the assumed form of $\Theta(\varphi)$ for $\varphi<0$ and so it only remains to describe how the integrals in (6) are approximated numerically. Some care must be exercised in formulating the numerical approximation of the integrals in (6). The solution method adopted here for the system of nonlinear equations is an iterative process which starts with an initial guess and with the assumption that the system is locally linear. The next guess in the iteration is calculated by inverting a numerically calculated Jacobian which in turn is calculated by finite differences. If the system were exactly linear then this would be a one-step solution method going straight from the initial guess to the (numerical) solution. The numerical stability of the inversion of the Jacobian is guaranteed if it is diagonally dominant, and it is with this constraint in mind that the numerical scheme is formulated. A further consideration is that the scheme should be accurate, so high-order integration (involving quadratic interpolations) is used rather than, for example, the trapezoidal rule.

Dealing with $\int_{0}^{\phi_{i}} \sin \theta d \varphi$ first, this integral can be written as

$$
\int_{0}^{\phi_{i}} \sin \theta d \varphi=\int_{0}^{\phi_{i-1}} \sin \theta d \varphi+\int_{\phi_{i-1}}^{\phi_{i}} \sin \theta d \varphi
$$

The second integral on the right hand side of the above equation is calculated by approximating $\sin \theta$ over $\left(\phi_{i-1}, \phi_{i}\right)$ by a parabola passing through $\sin \theta_{i-1}, \sin \theta_{i}$ and $\sin \theta_{i+1}$ and integrating the resulting quadratic exactly from $\phi_{i-1}$ to $\phi_{i}$. Thus $\int_{0}^{\phi_{i}} \sin \theta d \varphi$ is calculated sequentially. The use of the extra point $\phi_{i+1}$ which lies outside the range of integration was found to be necessary to ensure stability and accuracy of the overall method. If $\sin \theta$ was linearly interpolated over $\left(\sin \theta_{i-1}, \sin \theta_{i}\right)$ then the scheme was very diffusive with any waves present decaying with distance from the detachment point unless many points (over 100 per wavelength) were used. If $\sin \theta$ was approximated by a parabola through $\sin \theta_{i-2}, \sin \theta_{i-1}$ and $\sin \theta_{i}$ (that is, using only the interior points), then the resulting numerically calculated Jacobian was not diagonally dominant.

The Hilbert transform integral includes a singular point at $\varphi=\phi_{i}$. The integral can be written as

$$
f_{0}^{\infty} \frac{\theta}{\varphi-\phi_{i}} d \varphi=\int_{0}^{\phi_{i-1}} \frac{\theta}{\varphi-\phi_{i}} d \varphi+\int_{\phi_{i-1}}^{\phi_{i+1}} \frac{\theta}{\varphi-\phi_{i}} d \varphi+\int_{\phi_{i+1}}^{\infty} \frac{\theta}{\varphi-\phi_{i}} d \varphi
$$


The first and last integrals on the right hand side of the above equation are nonsingular and can be calculated accurately by assuming that $\theta$ is piecewise linear. The remaining integral contains the singular behaviour. To evaluate this integral, $\theta$ over the range $\left(\phi_{i-1}, \phi_{i+1}\right)$ is approximated by the parabola passing through $\theta_{i-1}, \theta_{i}$ and $\theta_{i+1}$. The resulting integral can be calculated exactly and is simply a linear combination of $\theta_{i-1}$, $\theta_{i}$ and $\theta_{i+1}$.

The resulting system of nonlinear equations is solved using a modified Powell hybrid method, as implemented in the NAG library subroutine C05NBF. In practice, $|\theta|$ is much less than 1 (typically less than 0.1 ) and thus the system of equations is close to linear, and hence the nonlinear equation solver converges quite rapidly. Acceptable grid-scale independence is achieved if there are more than 25 mesh points per wavelength and $\Delta \phi_{0}<0.01$. The effect of the truncation at $\phi_{N}$ is only felt within one wavelength of the truncation.

Having obtained $\theta(\phi)$ for $\phi>0$, the body and free surface in $(x, y)$ space can be obtained by integrating the equations

$$
\begin{aligned}
& \frac{\partial x}{\partial \phi}=e^{-\tau} \cos \theta \\
& \frac{\partial y}{\partial \phi}=e^{-\tau} \sin \theta
\end{aligned}
$$

from $\phi=-1$. To perform this integration numerically, the body $(-1<\phi<0)$ is discretised uniformly. The body angle $\Theta$ is known while $\tau$ needs to be calculated using a mixture of numerical and exact methods. More specifically,

$$
\tau=\frac{1}{\pi} f_{-1}^{0} \frac{\Theta}{\varphi-\phi_{i}} d \varphi+\frac{1}{\pi} f_{0}^{\infty} \frac{\theta}{\varphi-\phi_{i}} d \varphi .
$$

The first integral on the right-hand side of the above equation can be calculated exactly, while the second can be calculated using the methods already outlined. Once these integrals have been calculated, the right-hand sides of (9) are known functions of $\phi$, and it only remains to carry out a sequential integration to obtain the $(x, y)$ data. The integration is carried out over one interval from $\phi_{i}$ to $\phi_{i+1}$ by assuming that the right hand side is a parabola passing through the known values at $\phi_{i-1}, \phi_{i}$ and $\phi_{i+1}$. This method is used everywhere except for the first step from $\phi=-1$, where a linear form is used, and near values of $\phi$ where the right hand side contains an integrable singularity. In the latter case, Taylor series expansions of $x$ and $y$ near the critical value of $\phi$ are used to integrate up to and beyond this value of $\phi$. This special treatment near critical values of $\phi$ is necessary to ensure that there is grid-scale independence. The $(x, y)$ data provides a check of the numerical results since the elevation of the attachment point above the undisturbed free surface is given by $h=\left(1-q_{0}^{2}\right) / 2 \gamma$. Also, the linear theory wave-number is $\gamma$ which provides a further check of the results. 


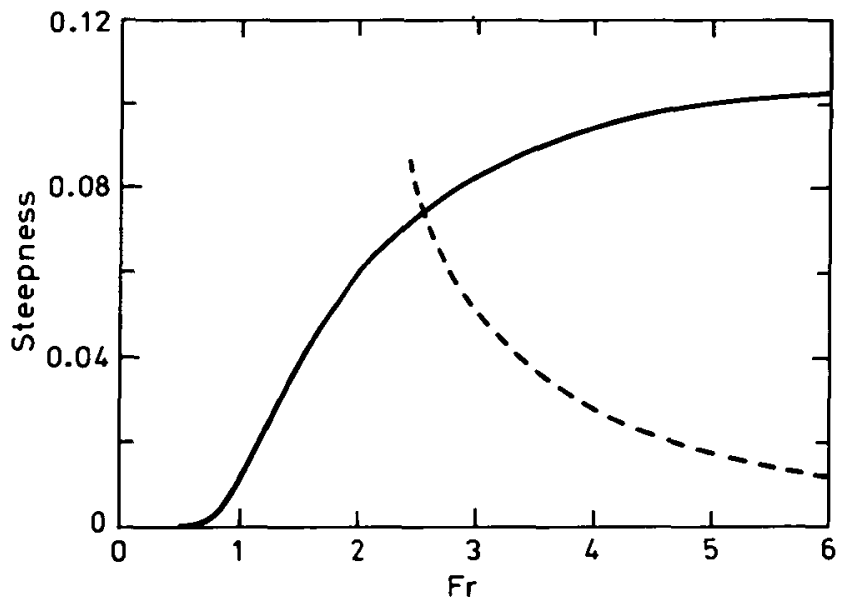

FIGURE 2. Steepness vs. draft-based Froude number for a rectangular stern, for both smooth (dashed) and stagnant (solid) attachment.

\section{Smooth-detachment results}

The numerical scheme is tested first on the simple case $\Theta(\varphi)=0$ for $\varphi<0$; that is, for a flat plate. This flat-plate problem with smooth detachment has been considered by Vanden-Broeck [5], who obtained an analytical relationship between the Froude number and the steepness of the waves (peak-to-trough height divided by the wavelength) far downstream from the stern. This degenerate stern geometry differs from others in that the Froude number, which is normally an output, can be specified a priori. In fact, in this special case only, $\gamma$ can be set to unity without loss of generality and the Froude number will then be determined by specifying the detachment velocity with $F r=\left(2 /\left(q_{0}^{2}-1\right)\right)^{1 / 2}$. Thus $q_{0}>1$ for real valued $F r$ or for ships with positive draft. Figure 2 shows (dashed curve) numerical results for the steepness versus $F r$ using the current method. These results agree quite well with the results of Vanden-Broeck [5]. Note that there are no solutions for $F r<2.26$, and that the steepness of the downstream waves vanishes as $F r \rightarrow \infty$. In fact, for large $F r$, the steepness is proportional to $\mathrm{Fr}^{-2}$. For all finite $\mathrm{Fr}$ above the critical value for the existence of solutions, there are waves of nonzero steepness present.

Of greater practical interest are those sterns that can generate solutions that have no waves far downstream. Such waveless solutions can be generated by a double-flap stern given by (see [2])

$$
\Theta= \begin{cases}\Theta_{0} & -1<\varphi<-b \\ \Theta_{1} & -b<\varphi<0\end{cases}
$$

again with smooth detachment. In this case, the Froude number cannot be specified $a$ priori, and is an output of the calculations. Figure 3 shows a contour plot of $F r$ and the 


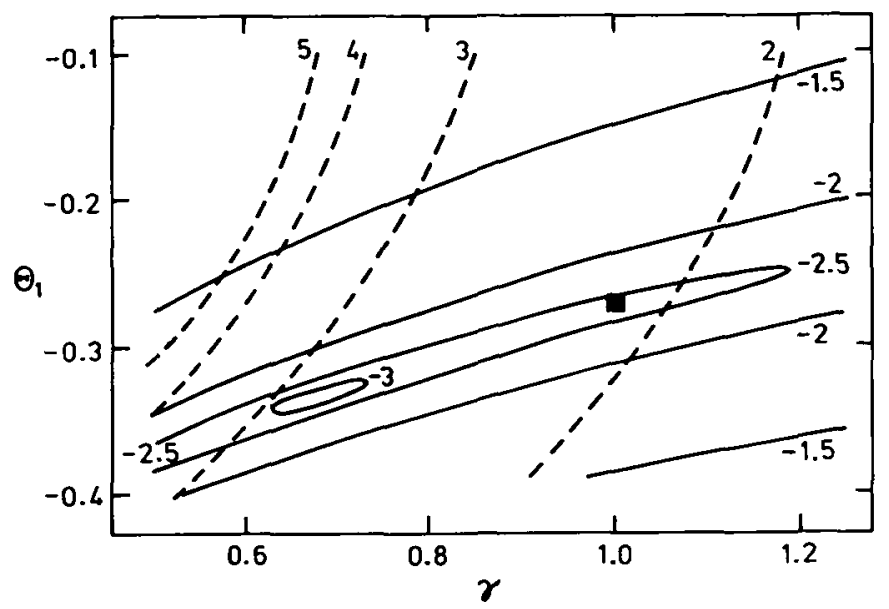

FIGURE 3. Contour plot of $F r$ (dashed contours, positive numbers) and $\log$ (steepness) (solid contours, negative numbers) in the $\left(\gamma, \Theta_{1}\right)$ plane, showing the "valley" of small-wave solutions. The solid square point lying in that valley is a waveless solution of Madurasinghe and Tuck [2].

logarithm of the steepness, as the angle $\Theta_{1}$ of the trailing edge and $\gamma$ are varied. The first flap angle $\Theta_{0}$ is held fixed at $\pi / 6$, and $b=0.25$. The filled square is a waveless solution found by Madurasinghe and Tuck, and lies in the narrow valley of solutions with waves of small steepness. The current method cannot calculate accurately the genuinely waveless solutions, but an examination of the solutions either side of the minimum (Figure 4) shows that the generated waves are almost out of phase, which is a necessary condition for the existence of a solution with no waves. The solutions are not exactly out of phase, since, for the waves to be visible, they can not be too close neighbours of the waveless solution. The current results are consistent with those of Madurasinghe and Tuck.

\section{Stagnant attachment results}

5.1. Rectangular stern Again, the numerical method of this paper is applied to the rectangular stern with $\Theta(\varphi)=\pi / 2$ for $-1<\varphi<0$. The attachment point $(\phi=0)$ is now assumed to be a stagnation point, which leads to the free surface and the stern being at right angles there (see [1]) and in fact, $\theta(\phi) \propto \sqrt{\phi}$ as $\phi \rightarrow 0^{+}$. This geometry has been considered by Vanden-Broeck and Tuck [6], where they also consider the more general case of sterns with $\Theta(\varphi)=\beta$, a constant, for $-1<\varphi<0$. Yeung [8] considered the unsteady development of the flow behind a rectangular stern, with the flow initially being at rest and accelerating smoothly to a steady velocity. For this geometry, there is a one-to-one relationship between the draft-based Froude number 


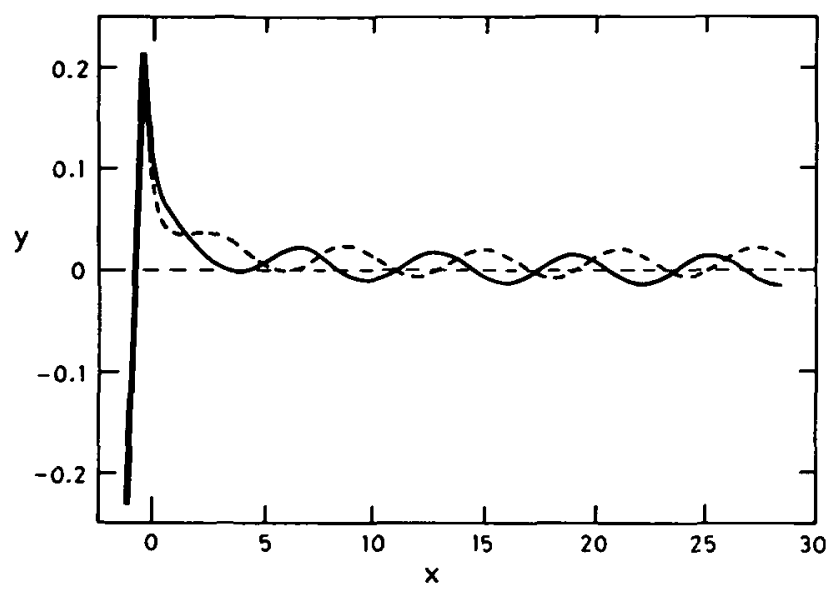

FIGURE 4. Neighbouring solutions from the previous figure showing the change in phase either side of the waveless solution.

and the input parameter $\gamma$. Figure 2 shows (solid curve) a plot of the steepness of the far downstream waves and the draft-based Froude number using the current method. The results are in good agreement with those of Vanden-Broeck, and confirm that there exists a flow of this type with waves for all $\mathrm{Fr}$. However, as $\mathrm{Fr} \rightarrow 0$, the wave amplitude becomes exponentially small [6]. Note also that for all $F r>0$, the downstream waves have a nonzero steepness, and hence the flow cannot be reversed to yield a splashless bow flow at any Froude number.

5.2. Bulbous sterns The results of this section are confined to the family of stern shapes considered by Tuck \& Vanden-Broeck [4] and Madurasinghe [1]. Tuck and Vanden-Broeck used an approach similar to the current paper where they found solutions for general sterns in this family that included waves, then manually adjusted a geometrical parameter of the stern until the resulting solution appeared to have no waves. Madurasinghe used a different approach, leaving one of the geometrical parameters free and specifying a wave-free solution a priori. Thus, his method would never find any solutions with waves. The stern shapes are members of the family

$$
\Theta= \begin{cases}\pi / 2 & -b<\phi<0 \\ A(\phi+1)(\phi+b)+\frac{\pi}{2}(\phi+1) /(1-b) & -1<\phi<-b \\ 0 & \phi<-1 .\end{cases}
$$

This stem consists of a vertical straight line segment and a rounded section joining it smoothly to the horizontal bottom. Setting $A<0$ yields bulbous sterns. Both Tuck and Vanden-Broeck [4] and Madurasinghe [1] found choices of $A$ and $b$ for which the resulting free surface was apparently waveless at special values of $\gamma$. The stern geometries in these cases were all bulbous in nature. Figure 5 is a graph of the stern 


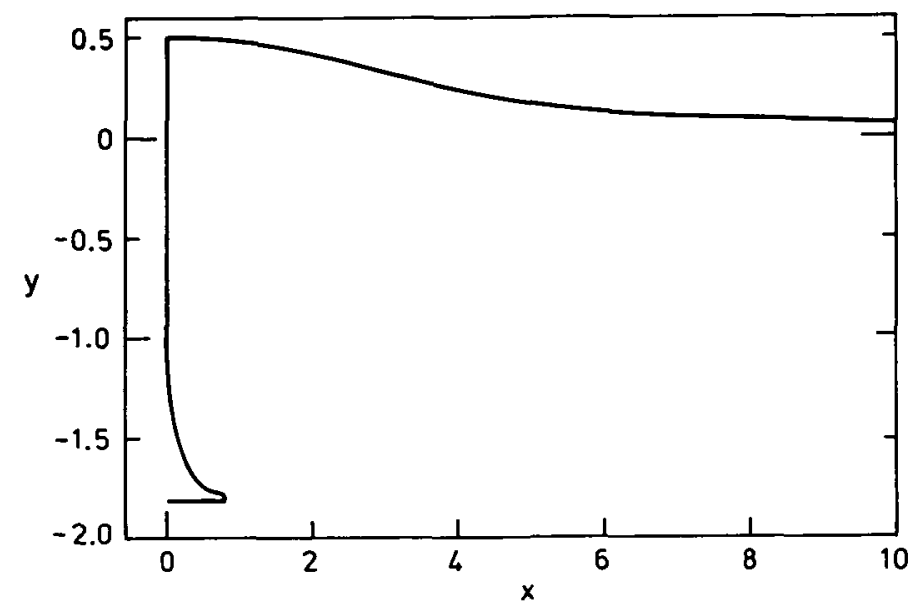

FIGURE 5. Stern and free surface profile of Tuck and Vanden-Broeck [4], recalculated using the method of this paper.

and free surface for $b=0.2, A=-14.01$ and $\gamma=1$, which corresponds to Figure 6 of Tuck and Vanden-Broeck [4] but now calculated using the current method. The results of the two separate calculations are in good agreement. The free surface would at first sight appear to be waveless, but on a closer examination of the numerical data, there are very small waves present and they have a steepness of $1.5 \times 10^{-3}$. The draft-based Froude number corresponding to this plot is $F r=0.74$, for which (see Figure 2, solid curve) a rectangular stern like that considered by Vanden-Broeck [5] would also generate waves of steepness $1.5 \times 10^{-3}$. Thus it would appear that not only has the bulb not eliminated the waves, it has not even had a significant impact on the wave amplitude associated with a rectangular stern at the same Froude number. The results of [4] show that wave steepness can be reduced at fixed $\gamma$, but the present results show that this is at the expense of reducing the Froude number.

A similar observation holds for the stern geometries considered by Madurasinghe [1]. In that paper, Madurasinghe identified curves in the $(\gamma, A)$ plane for fixed $b$ along which the sterns apparently generate no waves. Again, a close reexamination of the results along these curves using the current method reveals that there are waves present, but they have a very small magnitude. Figure 6 shows contours of the Froude number and the logarithm of the steepness of the waves in the $(\gamma, A)$ plane calculated using the current method. Superimposed on this plot is the " $C_{1}$ " curve of Madurasinghe [1] and the filled square is the small-wave solution of Tuck and Vanden-Broeck [4]. It is clear from this figure that increasing $\gamma$ decreases the steepness, as does decreasing $A$ (that is, making $A$ more negative). A striking feature of this figure is that the steepness and Froude number contours are nearly parallel. That is, as long as the Froude number is kept constant, the steepness would 


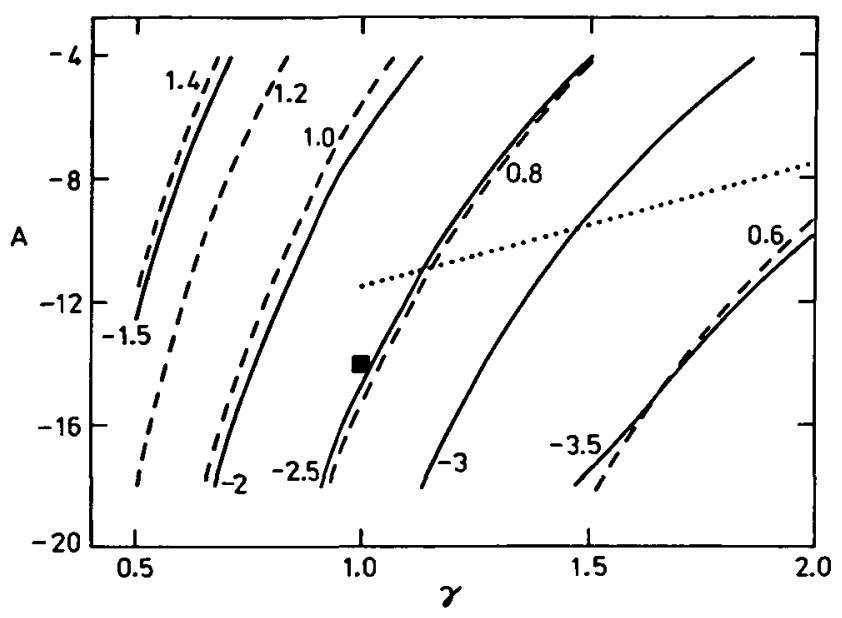

FIGURE 6. Contour plot of $\mathrm{Fr}$ (dashed contours, positive numbers) and $\log$ (steepness) (solid contours, negative numbers) in the $(\gamma, A)$ plane, showing also the $C_{1}$ (short-dash line) "waveless" solutions of Madurasinghe [1] and the (solid square point) "waveless" solution of Tuck and Vanden-Broeck [4].

appear to be almost independent of the stern's shape. Thus the small-wave solutions occur in a region of parameter space where all sterns make small waves, and the steepness of the waves made by all such sterns is essentially the same as that made by a rectangular stern at the same draft-based Froude number.

5.3. Bodies with smaller waves The family of sterns considered in the previous section allowed only strictly backward-pointing bulbs; that is, the ship lay entirely above the upstream draft. In this section, a more general family is considered that allows downward and even forward-pointing bulbs. These bodies consist of straight line segments connected by rounded comers. The family of stern shapes is given by

$$
\Theta(\varphi)= \begin{cases}\Theta_{1} & -1<\varphi<\phi_{1} \\ \Theta_{1}+\left(\Theta_{2}-\Theta_{1}\right) \frac{\varphi-\phi_{1}}{\phi_{2}-\phi_{1}} & \phi_{1}<\varphi<\phi_{2} \\ \Theta_{2} & \phi_{2}<\varphi<\phi_{3} \\ \Theta_{2}+\left(\pi / 2-\Theta_{2}\right) \frac{\varphi-\phi_{3}}{\phi_{4}-\phi_{3}} & \phi_{3}<\varphi<\phi_{4} \\ \pi / 2 & \phi_{4}<\varphi<0 .\end{cases}
$$

Thus this family is continuous on $(-1,0)$, and hence the corresponding stern will have no sharp corners except at $\phi=-1$. There are a total of six parameters that need to be specified in the above family.

Figure 7 shows a contour plot of the logarithm of the steepness and the draftbased Froude number, as the geometry and $\gamma$ are varied. The vertical axis in this plot corresponds to shifting the trailing edge (the $\left(\phi_{3}, \phi_{4}\right)$ section). In this figure, 


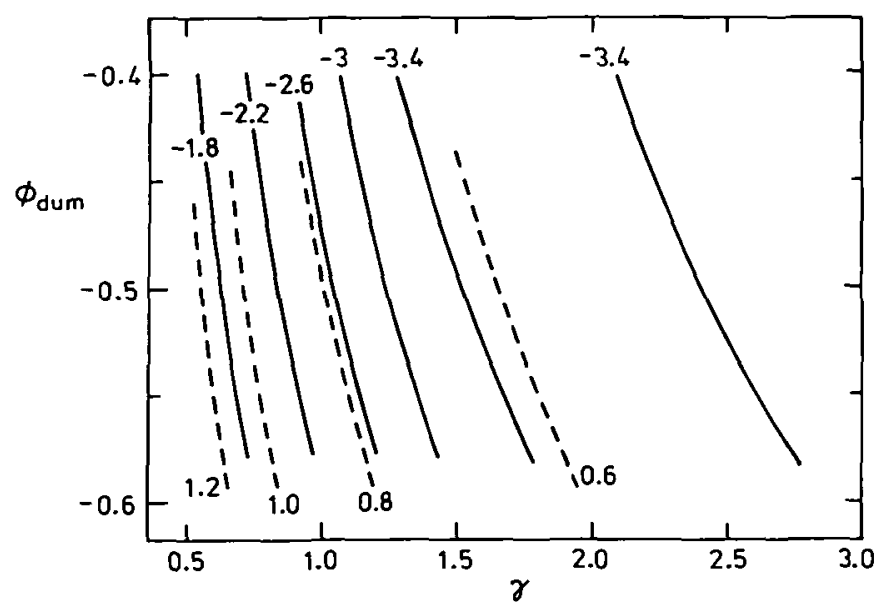

FIGURE 7. Contour plot of $F r$ (dashed contours, positive numbers) and $\log$ (steepness) (solid contours, negative numbers $)$ in the $\left(\gamma, \phi_{\text {dum }}\right)$ plane, with $\Theta_{0}=0$ and $\Theta_{1}=\pi$.

$\phi_{1}=-.9, \phi_{2}=-.8, \phi_{3}=\phi_{\mathrm{dum}}-.05, \phi_{4}=\phi_{\mathrm{dum}}+.05, \Theta_{1}=0$ and $\Theta_{2}=\pi$ and hence all sterns corresponding to this plot have backward-pointing bulbs with no downward component. This figure is similar to those of the previous section, in that the steepness and $F r$ contour lines are largely parallel, supporting the earlier conclusion that backward-pointing bulbs have little effect on wave steepness.

Figure 8 shows again a contour plot of the logarithm of the steepness and the draftbased Froude number as the geometry and $\gamma$ are varied. In this figure, $\Theta_{1}=-\pi / 2$ and $\Theta_{2}=0$ with the other parameters being the same as for Figure 7, and so all sterns of this plot have a downward-pointing bulb of varying width. The first thing to notice about this plot is that, unlike that of the previous figure with the backward-pointing bulb, the contour lines of the Froude number and the steepness are not parallel. This means that the changing geometry is having some effect on the downstream wave steepness. Figure 9 shows the stern and free surface profile for $F r=3.15$ and the corresponding free surface profile for a rectangular stern at the same Froude number. At this $F r$, a rectangular stern generates waves with steepness 0.0855 , whereas the stern with the downward-pointing bulb (which is quite huge and unrealistic, extending as it does to a depth more than 12 times the draft) yields waves with steepness 0.0119 . It is clear that the addition of the downward-pointing bulb has had a dramatic effect on the downstream wave steepness, reducing it by a factor of 7.2, although it has still not eliminated the waves entirely. 


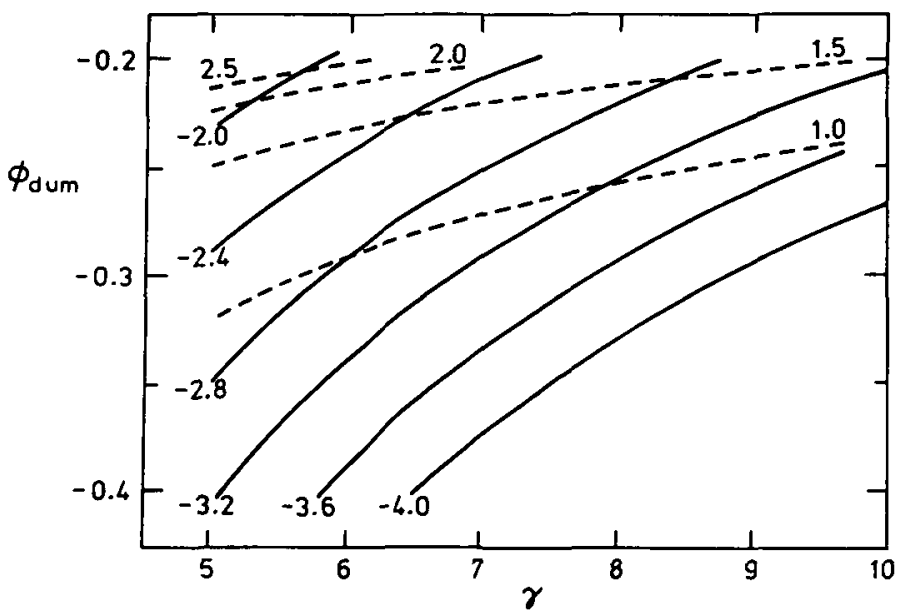

FIGURE 8. Contour plot of $F r$ (dashed contours, positive numbers) and $\log$ (steepness) (solid contours, negative numbers) in the $\left(\gamma, \phi_{\text {dum }}\right)$ plane, with $\Theta_{0}=-\pi / 2$ and $\Theta_{1}=0$.

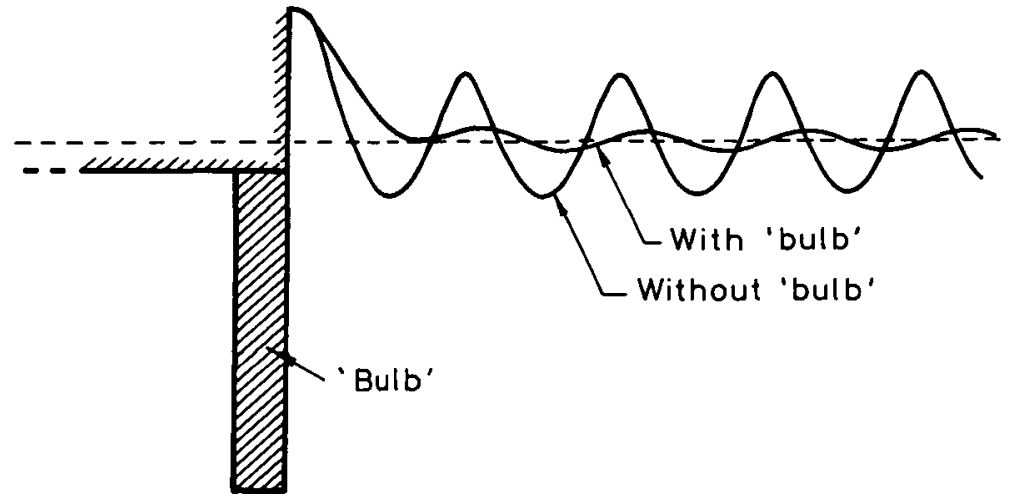

FIGURE 9. Comparison between the waves generated by a rectangular stern of draft $D$, and the (7 times smaller) waves generated at the same Froude number $F r=3.15$ (based on $D$ ) by a stern with a large rectangular downward projection at its aft end, of dimensions approximately length $20 D$ by depth $12.5 D$.

\section{Summary and conclusions}

This paper has reported the results of a new numerical method for calculating the free surface profile behind a semi-infinite stern-like body with an arbitrarily shaped section where the body ends. When the flow detaches smoothly from the body at a transom, the current results confirm the existence of special stern geometries which generate no waves. For those flows that rise to a stagnation point at detachment, the current results confirm the results (with waves) for rectangular sterns, but contradict the existence of previously published waveless solutions. More than that, the results 
suggest that (conventional) backward-pointing bulbs, previously thought to have beneficial effects on reducing wave steepness, in fact have no significant effect at all on the amplitude of the downstream waves, when compared for example with a bulb-less rectangular stern at the same draft-based Froude number. However, some members of another family that allows (unrealistically large) downward-pointing bulbs, while not eliminating waves entirely, do significantly reduce the magnitude of far downstream waves. A possible physical reason for the theoretical success of the downward bulbs in reducing wave height is that this very large appendage is acting like a twodimensional ship in its own right, with a length $L(L \approx 20 D$ in Figure 9) capable of yielding its own "bow-stern" wave cancellation, and that the nonzero but small draft $D$ far upstream is not playing a very important role in determining the free surface behaviour behind the ship.

\section{References}

[1] M. A. Madurasinghe, "Splashless ship bows with stagnant attachment", J. Ship Res. 32 (1988) 194-202.

[2] M. A. Madurasinghe and E. O. Tuck, "Ship bows with continuous and splashless flow attachment", J. Austral. Math. Soc. Ser. B 27 (1986) 442-452.

[3] E. O. Tuck, "Ship-hydrodynamic free-surface problems without waves", J. Ship Res. 35 (1991) 227-287.

[4] E. O. Tuck and J.-M. Vanden-Broeck, "Spashless bow flows in two dimensions?", Proc. 15th Symp. Naval Hydrodynamics, Hamburg (1984) 293-301.

[5] J.-M. Vanden-Broeck, "Nonlinear stern waves", J. Fluid Mech. 96 (1980) 603-611.

[6] J.-M. Vanden-Broeck and E. O. Tuck, "Computation of near-bow or stem flows, using series expansion in the Froude number", Proc. 2nd International Conf. on Numerical Ship Hydrodynamics, Berkeley (1977) 293-301.

[7] J.-M. Vanden-Broeck and E. O. Tuck, "Waveless free-surface pressure distributions", J. Ship Res. 29 (1985) 151-158.

[8] R. W. Yeung, "Nonlinear bow and stern waves-inviscid and viscous solutions", in Mathematical Approaches in Hydrodynamics (ed. T. Miloh), M. Tulin anniversary volume, (S.I.A.M., 1991) 349-369. 